\title{
POPULATIONS OU ESPÈCES ?
}

\author{
Recherches sur la signification de la transmission \\ de Trématodes Lepocreadiinae (T. Odhner, 1905) \\ dans deux écosystèmes marins.
}

\author{
P. BARTOLI*
}

RÉSUMÉ. Deux cercaires ophthalmotrichocerques, très semblables au point de vue de leurs caractères morpho-anatomiques, parasitent deux espèces distinctes de Prosobranches marins : Sphaeronassa mutabilis et Amyclina corniculum. Le développement de ces deux cercaires a été suivi jusqu'au stade adulte correspondant.

Les cycles de vie de ces deux lignées de Trématodes s'avèrent différents non seulement au niveau du premier hôte intermédiaire, mais aussi des deuxièmes hôtes (Lamellibranches) ; seuls les hôtes définitifs sont communs (Sparus auratus, Pagellus erythrinus, P. mormyrus). Par ailleurs, les endémiotopes dans lesquels se déroulent les cycles de ces deux lignées, sont fondamentalement différents : plages de sables fins, entre 0 et $10 \mathrm{~m}$ de profondeur (lignée issue de $S$. mutabilis); aires vaseuses de mode calme à la périphérie de l'herbier de Cymodocea nodosa, entre 0 et $10 \mathrm{~cm}$ de profondeur (lignée $A$. corniculum).

$\mathrm{Au}$ point de vue biométrique, plusieurs différences significatives sont notées entre les stades larvaires correspondants de ces deux lignées.

En dehors de ces divergences, presque tous les caractères morphologiques, anatomiques et biologiques sont identiques chez les divers stades de ces deux lignées.

Il s'agit là probablement de deux populations d'une même espèce, Lepocreadium pegorchis. Celles-ci se mélangeraient chez les Poissons hôtes définitifs qui passent fréquemment d'un écosystème dans l'autre. Si cette hypothèse se trouvait vérifiée on serait alors en présence d'un exemple remarquable de vicariance écologique.

\section{Populations or species ? Research on the transmission of Lepocreadiinae (T. Odhner, 1905) (Trematoda) in two marine ecosystems.}

SUMMARY. Two ophthalmotrichocercous cercariae, very similar as concerns their morphological and anatomical characteristic, parasitize two distincts species of marine Prosobranches : Sphaeronassa mutabilis and Amyclina corniculum. The development of these two cercariae has been followed through the corresponding adult stages.

The life cycles of these two lines of Trematodes were shown to be different not only for the first intermediate host but also for the second hosts (Pelecypods); only the final hosts are shared (Sparus auratus, Pagellus erythrinus and P. mormyrus). Additionally the endemiotops (where the life cycle develops) are fundamentally different. Beaches of fine sand with a depth of 0 to $10 \mathrm{~m}$ are the endemiotop of the line arising from $S$. mutabilis whereas hydrodynamically calm

* Laboratoire de Zoologie Marine, Faculté des Sciences et Techniques de Saint-Jérôme, Université d'Aix-Marseille III, F 13397 Marseille Cedex 13.

Accepté le 5 octobre 1982 . 
muddy biotops (depth between 0 and $10 \mathrm{~cm}$ ) found at the periphery of prairies of Cymodocea nodosa are the endemiotop of the line arising from $A$. corniculum.

A biometrical study of every larval stage shows significant differences between those two lines. In spite of these differences, the most part of morphological, anatomical and biological characteristics are the same among the various stages of the two lines.

So we think the two lines could be, in fact, two populations of the same species, Lepocreadium pegorchis M. Stossich, 1900 which are found together into the definitive hosts, because these fishes are living into the ecosystems characteristic of the two lines. If so, we should be faced to a noteworthly example of ecological vicariance.

Le Trématode Lepocreadium pegorchis M. stossich, 1900 parasite à l'état adulte divers Poissons néritiques de Méditerranée. Son cycle biologique hétéroxène comporte un premier hôte intermédiaire Sphaeronassa mutabilis (L.) (syn. : Nassarius mutabilis) (Mollusque Prosobranche) ( $f i g . ~ 1, A)$ et divers deuxièmes hôtes qui sont tous des Mollusques Lamellibranches (P. Bartoli, 1966 et 1967).

La cercaire de $L$. pegorchis, parasite de S. mutabilis, appartient au groupe ophthalmotrichocerque; elle est caractérisée par une queue de longueur nettement plus courte que celle du corps ce qui la distingue d'emblée des autres cercaires de ce groupe.

La découverte sur les côtes de Provence d'une cercaire morphologiquement très semblable à celle parasitant $S$. mutabilis mais issue d'une autre espèce de Prosobranche, Amyclina corniculum (Olivi), (fig. 1, B) nous a conduit à rechercher s'il s'agissait ou non de la même espèce de Trématode. On connât en effet plusieurs cas chez les Digènes, où des cercaires très proches sur le plan morpho-anatomique, s'avèrent correspondre en fait à des adultes différents, donc à des espèces distinctes. Ainsi, dans la sous-famille des Lepocreadiinae à laquelle appartiennent ces deux cercaires méditerranéennes, H. W. Stunkard (1980) a démontré l'existence, sur les côtes de Floride, de deux espèces congénériques sympatriques, très semblables à chacun des stades de leur cycle de vie. Chez ces deux Lépocréadiens, Neopechona pyriforme (Linton, 1900) Stunkard, 1969 et Neopechona cablei Stunkard, 1980, les adultes parasitent une même espèce de Poissons, Stenopus chrysops; par contre, les cercaires, très ressemblantes, parasitent des Prosobranches différents : Anachis avara dans le premier cas, Mitrella lunata dans le second.

Dans l'exemple méditerranéen qui nous occupe, on doit se demander si ces deux cercaires issues de Mollusques différents, ne correspondent pas elles aussi à deux espèces distinctes.

\section{I - Comparaison des cycles biologiques}

Dans la nature, les cercaires parasites de Sphaeronassa mutabilis évoluent en métacercaires chez un grand nombre d'espèces de Lamellibranches; par contre, celles issues de Amyclina corniculum se rencontrent chez trois espèces seulement (tableau I). Les adultes naturels correspondants parasitent diverses espèces de Sparidés. Dans les deux cas, il s'agit de Poissons de mêmes espèces. 


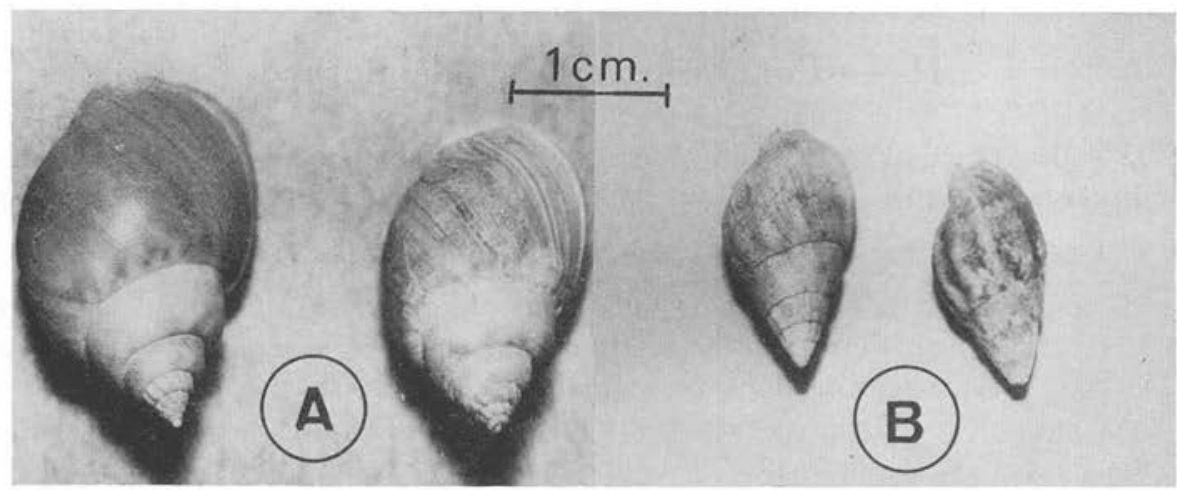

FIG. I. - Premiers hôtes intermédiaires (Mollusques adultes). A : Sphaeronassa mutabilis (L.) ; B : Amyclina corniculum (Olivi).

TABleau I. - Comparaison des cycles biologiques (hôtes naturels).

Lepocreadium pegorchis

M. Stossich, 1900

Premier hôte intermédiaire

Deuxièmes hôtes intermédiaires
Nassidae
Sphaeronassa mutabilis (L.)

Cardiidae:

Acanthocardia tuberculata (L.) Parvicardium ovale (Sowerby)

Veneridae:

Venus gallina L.

Mactridae:

Mactra corallina $\mathrm{L}$.

Spisula subtruncata Da Costa

Donacidae:

Donax trunculus L.

Donax semistriatus Poli

Tellinidae:

Tellina nitida Poli

Pandoridae:

Pandora flexuosa Sowerby
Nassidae:

Amyclina corniculum (Olivi)

Cardiidae:

Cerastoderma glaucum Poiret

Veneridae :

Venus verrucosa $\mathrm{L}$.

Venerupis aurea (Gmelin)

Hôtes définitifs

Sparidae:

Sparus auratus

Pagellus erythrinus

Pagellus mormyrus

Sparidae:

Sparus auratus

Pagellus erythrinus

Pagellus mormyrus 


\section{II - Comparaison des endémiotopes}

L'endémiotope d'un parasite correspond au biotope dans lequel toutes les conditions sont réunies pour que son cycle biologique se déroule de façon complète. A - Endémiotope du parasite de Sphaeronassa mutabilis (fig. 2, A)

Il correspond à la biocénose de sables fins superficiels (S.F.S.) de J. M. Pérès et J. Picard (1964). Cette biocénose s'étend sur toute la longueur des plages de sables fins. Dans le golfe de Marseille, au niveau de la Plage du Prado, ces sables fins ont une bathymétrie comprise entre 0 et $8 \mathrm{~m}$ ou $9 \mathrm{~m}$ (J. Picard, 1965). L'hydrodynamisme y est souvent important. La température de l'eau ne subit pas de brusques variations.

Le premier hôte intermédiaire (Sphaeronassa mutabilis) et les divers deuxièmes hôtes (Donax trunculus, Acanthocardia tuberculata, etc.) sont des espèces étroitement associées à cette biocénose des sables fins superficiels. Le cycle de vie de Lepocreadium pegorchis est donc parfaitement adapté à cette biocénose.

\section{B - Endémiotope du parasite de Amyclina corniculum (fig. 2, B)}

Il est confiné dans les fonds de baie et dans les aires très protégées ; par voie de conséquence, ces zones sont souvent nitrophiles (faciès de pollution). Lorsque le substrat est sablo-vaseux, par exemple dans la lagune du Brusc (Var), l'endémiotope se situe à un niveau très légèrement supérieur à celui de l'herbier de Cymodocea nodosa ; il s'étale donc à la périphérie de la prairie. Cet endémiotope est ainsi limité à des aires très peu profondes (de 0 à $-10 \mathrm{~cm}$ ), souvent exondées en raison des marées barométriques ou du vent. L'hydrodynamisme est pratiquement nul. L'eau, peu renouvelée, subit de brusques variations de température, souvent de très grande amplitude.
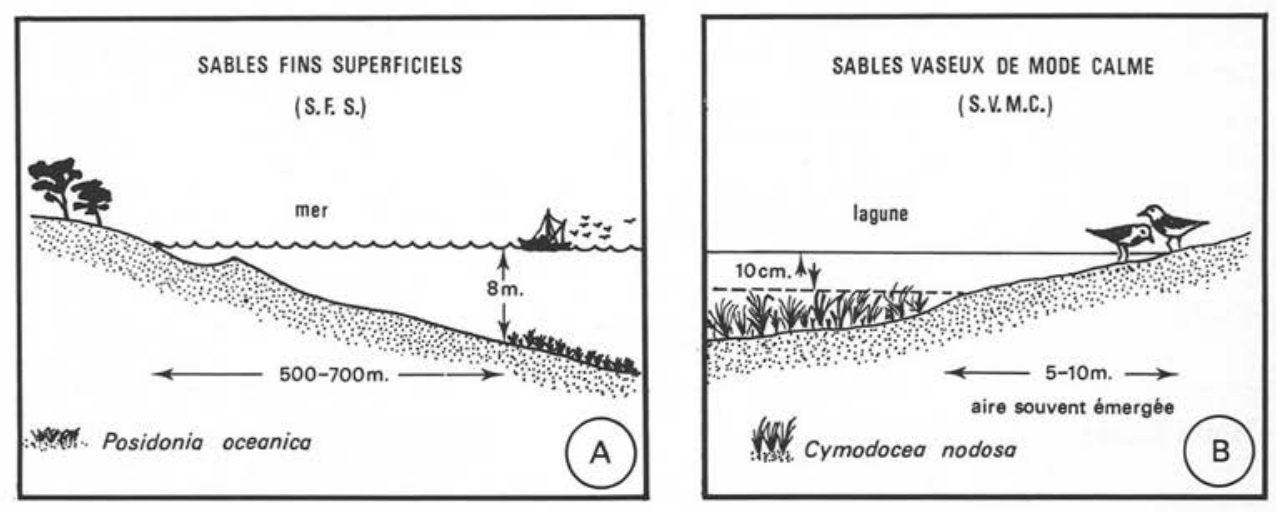

FIG. 2. - Comparaison des endémiotopes. A : endémiotope du parasite de Sphaeronassa mutabilis. B : endémiotope du parasite de Amyclina corniculum. 
Le premier hôte intermédiaire, Amyclina corniculum, est bien adapté à ce milieu confiné ; c'est une espèce indicatrice de pollution que l'on rencontre particulièrement dans la zone des algues nitrophiles (P. Mars, 1966). Parmi les deuxièmes hôtes intermédiaires, Venerupis aurea est une espèce caractéristique des sables vaseux de mode calme (SVMC) (J. Picard, 1965) ; Cerastoderma glaucum est inféodé aux milieux confinés, lagunaires (P. Mars, 1966; J, Picard, 1965). Dans ce cas encore, le cycle biologique du parasite est parfaitement adapté à ce type de milieu.

Les deux endémiotopes décrits ci-dessus n'interfèrent jamais l'un avec l'autre ; ils sont toujours très nettement séparés.

\section{III - Comparaison des caractères morpho-anatomiques}

Tous les stades naturels du cycle de vie de Lepocreadium pegorchis ont été récoltés dans la biocénose des sables fins superficiels (S.F.S.) du Golfe du Prado (Marseille). Avec les cercaires issues spontanément de Sphaeronassa mutabilis, nous avons obtenu à de nombreuses reprises les métacercaires chez des Lamellibranches indemnes et, avec celles-ci, les adultes correspondants chez des Dorades élevées artificiellement. De même, tous les stades naturels du cycle débutant chez Amyclina corniculum ont été trouvés dans la biocénose des sables vaseux de mode calme (S.V.M.C.) de la lagune du Brusc (Var). En utilisant les cercaires émises naturellement par A. corniculum nous avons obtenu à diverses reprises les métacercaires expérimentales puis les adultes correspondants.

Ainsi, dans les deux lignées, nous avons pu disposer d'un abondant matériel d'origine soit naturelle, soit expérimentale.

Les différents stades du cycle de vie ayant pour point de départ $S$. mutabilis ont déjà été décrits (P. Bartoli, 1967). En dehors de quelques nouvelles précisions, nous nous bornerons à comparer ces divers stades avec ceux de la lignée issue de A. corniculum.

Chaque parasite a été tué par la chaleur puis modérément aplati, fixé, coloré et monté entre lame et lamelle.

\section{A - Les rédies}

Dans chaque hôte, on distingue toujours deux types de rédies :

- des rédies de petite taille. Elles sont très semblables dans les deux lignées. Cependant, celles parasitant $S$. mutabilis ont des dimensions légèrement inférieures à celles infestant A. corniculum (tableau II; fig. 3).

- des rédies de grande taille. Dans ce cas encore, les grandes rédies rencontrées chez $S$. mutabilis sont un peu moins volumineuses que celles parasitant $A$. corniculum (tableau III; fig. 4).

Nous n'avons jamais observé de pore de naissance chez aucune rédie des deux lignées. Par contre, on trouve parfois de grosses rédies dont la région postérieure est 
Tableau II. - Mensurations comparées (en $\mu \mathrm{m}$ ) de deux échantillons de rédies de petites taille; le premier est constitué par des rédies parasites de Sphaeronassa mutabilis, le second par des rédies parasites de Amyclina corniculum.

\begin{tabular}{|c|c|c|c|c|c|c|}
\hline & \multicolumn{3}{|c|}{$\begin{array}{c}\text { Rédies } \\
\text { de Sphaeronassa mutabilis }\end{array}$} & \multicolumn{3}{|c|}{$\begin{array}{c}\text { Rédies } \\
\text { de Amyclina corniculum }\end{array}$} \\
\hline & $\begin{array}{l}\text { Varia- } \\
\text { tions }\end{array}$ & Moyenne & $\begin{array}{l}\text { Int. } \\
\text { conf. } \\
(1 \%)\end{array}$ & $\begin{array}{l}\text { Varia- } \\
\text { tions }\end{array}$ & Moyenne & $\begin{array}{l}\text { Int. } \\
\text { conf. } \\
(1 \%)\end{array}$ \\
\hline Longueur totale & $200-360$ & 269 & 41,4 & $292-416$ & 366,4 & 46,2 \\
\hline Largeur & $43-61$ & 52 & 5 & $53-80$ & 59,9 & 2,7 \\
\hline Longueur du pharynx & $35-52$ & 40 & 5,2 & $47-56$ & 52,7 & 1,14 \\
\hline Largeur du pharynx & $31-49$ & 38 & 5,8 & $46-53$ & 49 & 1 \\
\hline
\end{tabular}

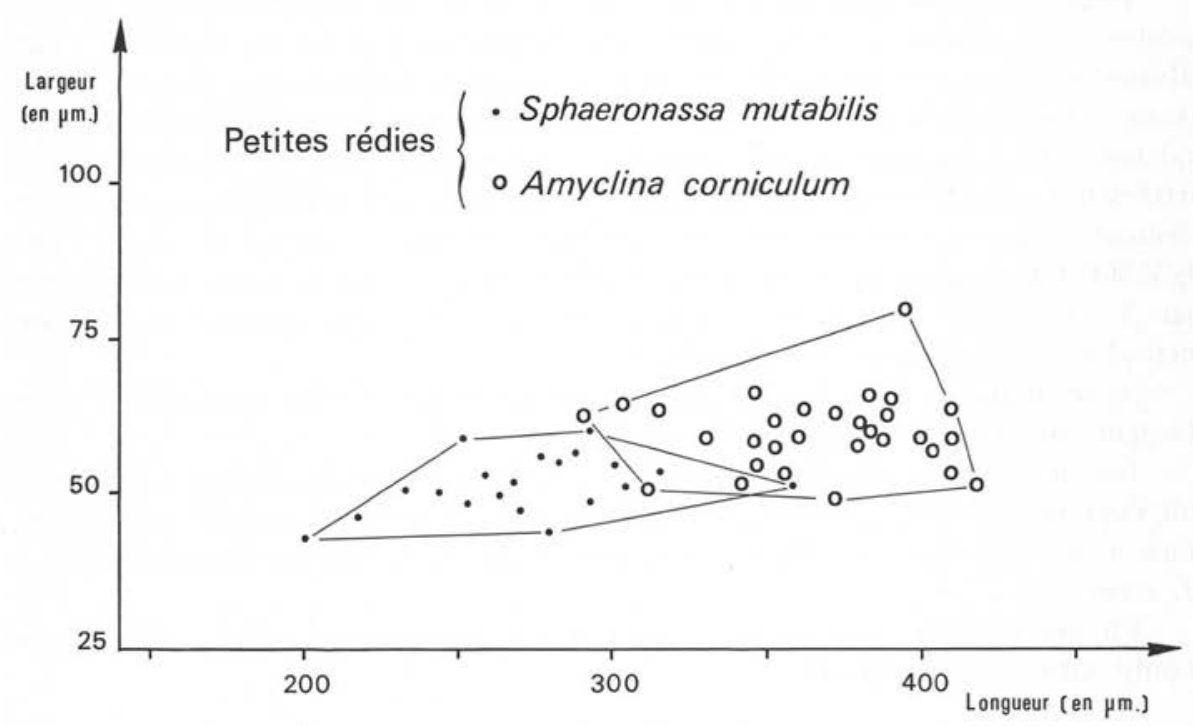

FIG. 3. - Les petites rédies parasitant Sphaeronassa mutabilis ont des dimensions inférieures à celles qui parasitent Amyclina corniculum.

lysée tandis que la partie antérieure est parfaitement vivante ; elles sont dépourvues de cercaires. Tout laisse supposer qu'il n'y a pas ponte mais libération des cercaires par destruction de la paroi de la rédie.

\section{B - Les cercaires}

Seules les cercaires émises naturellement sont prises en considération dans ce travail (fig. 5). 
TABLEAU III. - Mensurations comparées (en $\mu \mathrm{m}$ ) de deux échantillons composés chacun de 30 rédies de grande taille; le premier est constitué par des rédies parasites de Sphaeronassa mutabilis, le second par des rédies parasites de Amyclina corniculum.

\begin{tabular}{|c|c|c|c|c|c|c|}
\hline & \multicolumn{3}{|c|}{$\begin{array}{c}\text { Rédies } \\
\text { de Sphaeronassa mutabilis }\end{array}$} & \multicolumn{3}{|c|}{$\begin{array}{c}\text { Rédies } \\
\text { de Amyclina corniculum }\end{array}$} \\
\hline & $\begin{array}{l}\text { Varia- } \\
\text { tions }\end{array}$ & Moyenne & $\begin{array}{l}\text { Int. } \\
\text { conf. } \\
(1 \%)\end{array}$ & $\begin{array}{l}\text { Varia- } \\
\text { tions }\end{array}$ & Moyenne & $\begin{array}{l}\text { Int. } \\
\text { conf. } \\
(1 \%)\end{array}$ \\
\hline Longueur totale & $693-1012$ & 835 & 41 & $879-1279$ & 1080 & 49 \\
\hline Largeur & $101-160$ & 128 & 7,8 & $117-197$ & 160,9 & 10,8 \\
\hline Longueur du pharynx & $35-54$ & 43,4 & 2,4 & $52-80$ & 62 & 3,1 \\
\hline Largeur du pharynx & $31-61$ & 42,2 & 3,7 & $47-78$ & 58,7 & 3,4 \\
\hline Longueur de la queue & $133-256$ & 185,8 & 16,6 & $139-304$ & 222,3 & 47,4 \\
\hline
\end{tabular}

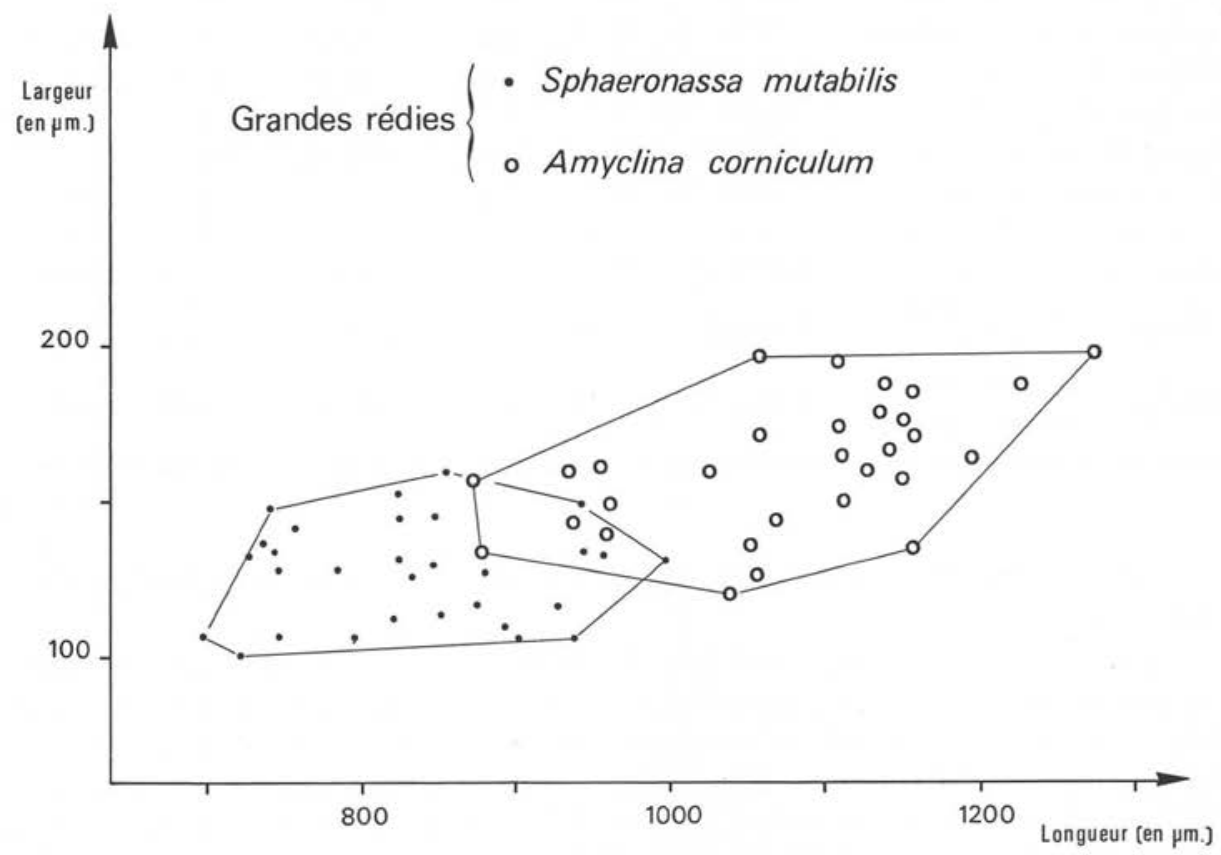

FIG. 4. - Les grandes rédies parasitant Sphaeronassa mutabilis ont des dimensions inférieures à celles qui parasitent Amyclina corniculum.

Les mensurations des cercaires parasites de Sphaeronassa mutabilis sont généralement inférieures à celles des cercaires issues de Amyclina corniculum (tableau IV) ; pour plusieurs organes, en particulier pour le pharynx, les différences sont significatives au seuil de $1 \%$. 
TABLEAU IV. - Mensurations comparées (en $\mu \mathrm{m}$ ) de deux échantillons composés chacun de 30 cercaires émises spontanément par leur hôte.

\begin{tabular}{|c|c|c|c|c|c|c|}
\hline & \multicolumn{3}{|c|}{$\begin{array}{c}\text { Cercaires } \\
\text { de Sphaeronassa mutabilis }\end{array}$} & \multicolumn{3}{|c|}{$\begin{array}{c}\text { Cercaires } \\
\text { de Amyclina corniculum }\end{array}$} \\
\hline & $\begin{array}{l}\text { Varia- } \\
\text { tions }\end{array}$ & Moyenne & $\begin{array}{l}\text { Int. } \\
\text { conf. } \\
(1 \%)\end{array}$ & $\begin{array}{l}\text { Varia- } \\
\text { tions }\end{array}$ & Moyenne & $\begin{array}{l}\text { Int. } \\
\text { conf. } \\
\text { (1 \%) }\end{array}$ \\
\hline Longueur totale & $206-256$ & 235 & 5,4 & $202-284$ & 247 & 9,2 \\
\hline Largeur totale & $93-111$ & 105 & 4,9 & $91-141$ & 110 & 6,5 \\
\hline Longueur préacétabulaire & $82-108$ & 94 & 2,3 & $87-135$ & 110 & 1,6 \\
\hline Longueur postacétabulaire & $87-117$ & 102 & 7,4 & $78-119$ & 97 & 4,8 \\
\hline Longueur ventouse orale & $42-54$ & 49 & 2,9 & $44-57$ & 52 & 1,6 \\
\hline Largeur ventouse orale & $39-53$ & 42 & 2,7 & $44-60$ & 49 & 2,2 \\
\hline Longueur ventouse ventrale & $32-45$ & 39 & 3,4 & $30-53$ & 39 & 2,5 \\
\hline Largeur ventouse ventrale & $39-52$ & 46 & 3,3 & $36-57$ & 51 & 2,6 \\
\hline Longueur du pharynx & $25-30$ & 27 & 1,4 & $30-39$ & 36 & 1,4 \\
\hline Largeur du pharynx & $18-24$ & 21 & 1,3 & $22-29$ & 25 & 0,9 \\
\hline Longueur de la queue & $145-176$ & 166 & 6,1 & $176-208$ & 191 & 4,3 \\
\hline Largeur de la queue & $20-26$ & 23 & 1,5 & $22-30$ & 25 & 1,2 \\
\hline Distance entre deux rames & $6-8$ & 6,9 & 0,4 & $7-8$ & 7,3 & 0,2 \\
\hline Rapport ventousaire & $1.07-1.52$ & 1,27 & 0,11 & $0,94-1,76$ & 1,35 & 0,08 \\
\hline Rapport $\frac{\text { ventouse orale }}{\text { pharynx }}$ & $1,54-2,07$ & 1,80 & 0,13 & $1,28-1,70$ & 1,46 & 0,04 \\
\hline Rapport $\frac{\text { longueur corps }}{\text { longueur queue }}$ & $1,30-1,58$ & 1,42 & 0,07 & $1,09-1,50$ & 1,28 & 0,12 \\
\hline
\end{tabular}

Les cercaires de $S$. mutabilis et de A. corniculum possèdent les caractères communs suivants :

- glandes céphaliques : elles sont très développées (fig. 6). Leurs corps cellulaires sont situés sur les côtés et légèrement en avant de la ventouse ventrale ; les canalicules longent le pharynx, passent entre celui-ci et la tache oculaire puis cheminent dorsalement le long de la ventouse orale. Quatre à cinq paires de canalicules se détachent de l'ensemble des autres canaux et viennent déboucher en avant du pharynx; ce sont des glandes pharyngiennes. Leurs corps cellulaires sont mélangés à ceux des glandes céphaliques.

- taches oculaires : elles sont très développées; on y compte une ou deux lentilles. De très nombreux granules pigmentaires sont épars dans le parenchyme; on en retrouve dans la totalité de l'espace préacétabulaire.

- appareil excréteur : les cellules à flamme vibratile sont disposées entre elles selon la formule : $2[(5 ?+5+5)+(5+5+5)]$. La grappe antérieure paraît incomplète ; 

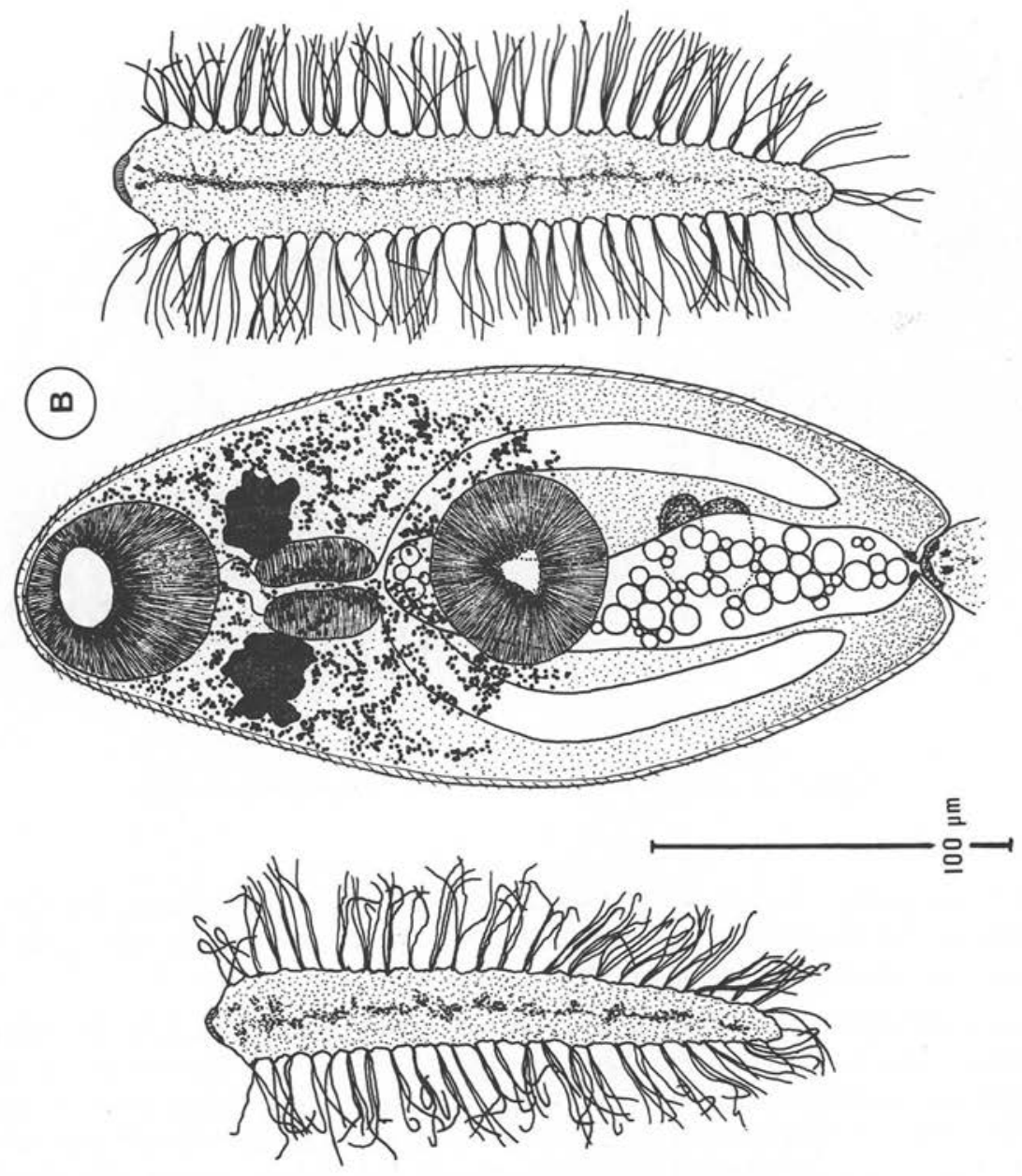

(4)

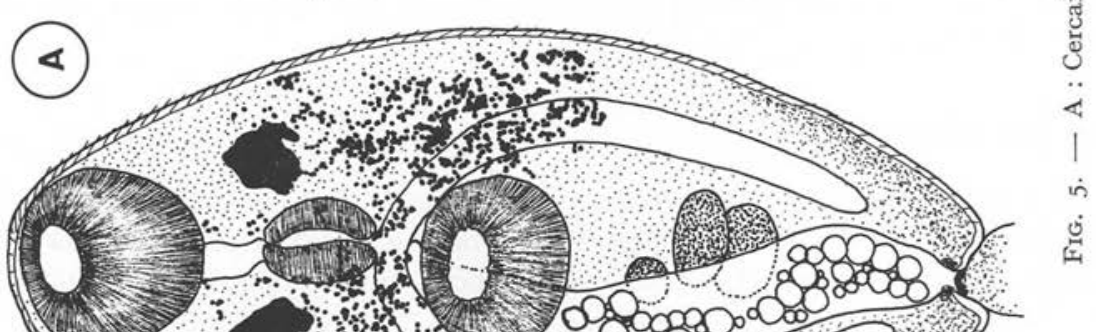
Fin:

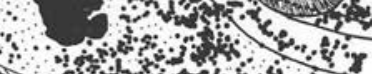
ats 

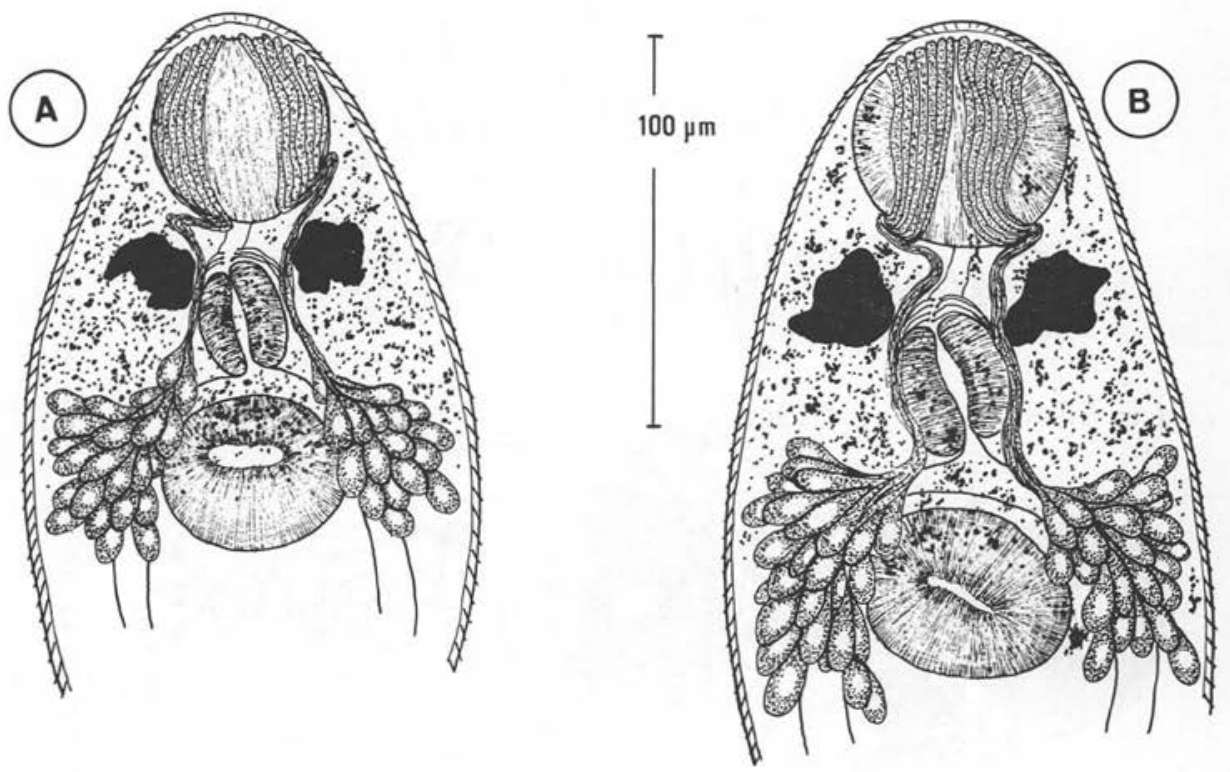

FIG. 6. - Glandes céphaliques et pharyngiennes. A : cercaire émise par Sphaeronassa mutabilis ; B : cercaire issue de Amyclina corniculum.

elle est généralement composée de trois ou de quatre protonéphridies. La vessie excrétrice est tubulaire; son extrémité antérieure atteint toujours le niveau de la ventouse ventrale et peut même la dépasser en avant (fig. 5).

- Queue : la longueur de la queue est invariablement inférieure à celle du corps. Par contre, c'est l'inverse chez les cercaires de la plupart des Lepocreadiinae. Le nombre de rames sétigères est assez variable chez les Lepocreadiinae; ici, on en compte 26 ou 27 paires, exceptionnellement 25 ou 28. Chaque rame est composée généralement de 4 ou 5 soies, rarement de 6 . Le bouquet terminal, impair, comprend habituellement de 6 à 8 soies.

\section{C - Les métacercaires}

Les dimensions des métacercaires de la lignée Sphaeronassa mutabilis sont inférieures à celles des métacercaires appartenant à la lignée Amyclina corniculum $($ tableau $V)$; dans la plupart des cas les différences sont significatives au seuil de $1 \%$.

\section{D - Les adultes}

Chez les adultes des deux lignées, les dimensions de chaque organe varient dans d'assez larges limites. Cependant, pour beaucoup d'entre eux, les différences entre 
TAbleau V. - Mensurations comparées (en $\mu \mathrm{m}$ ) de deux échantillons composés chacun de 70 métacercaires développées expérimentalement chez Cerastoderma glaucum. Le premier lot appartient à la lignée Sphaeronassa mutabilis, le second à la lignée Amyclina corniculum.

\begin{tabular}{|c|c|c|c|c|c|c|}
\hline & \multicolumn{3}{|c|}{$\begin{array}{l}\text { Lignée } \\
\text { Sphaeronassa mutabilis }\end{array}$} & \multicolumn{3}{|c|}{$\begin{array}{l}\text { Lignée } \\
\text { Amyclina cornicuulm }\end{array}$} \\
\hline & $\begin{array}{l}\text { Varia- } \\
\text { tions }\end{array}$ & Moyenne & $\begin{array}{l}\text { Int. } \\
\text { conf. } \\
(1 \%)\end{array}$ & $\begin{array}{l}\text { Varia- } \\
\text { tions }\end{array}$ & Moyenne & $\begin{array}{l}\text { Int. } \\
\text { conf. } \\
(1 \%)\end{array}$ \\
\hline Longueur totale & $152-278$ & 208 & 9,4 & $200-370$ & 258 & 13,6 \\
\hline Largeur totale & $74-124$ & 93,2 & 3,6 & $85-148$ & 109 & 5,8 \\
\hline Longueur préacétabulaire & $65-122$ & 86,3 & 3,8 & $85-176$ & 115,4 & 7,5 \\
\hline Longueur postacétabulaire & $54-115$ & 81,2 & 5,2 & $74-139$ & 100,2 & 5 \\
\hline Longueur ventouse orale & $31-51$ & 42,8 & 2,10 & $45-59$ & 51,2 & 1,7 \\
\hline Largeur ventouse orale & $33-51$ & 42 & 1,6 & $44-71$ & 54,9 & 2,4 \\
\hline Longueur ventouse ventrale & $28-49$ & 38,8 & 1,6 & $34-56$ & 43,4 & 2 \\
\hline Largeur ventouse ventrale & $29-51$ & 41,3 & 1,6 & $35-63$ & 48 & 2,8 \\
\hline Longueur pharynx & $19-32$ & 25,7 & 1 & $27-38$ & 32,7 & 1,1 \\
\hline Largeur pharynx & $17-25$ & 20,4 & 0,6 & $19-38$ & 27 & 2 \\
\hline Rapport ventousaire & $0,9-1,3$ & 1,1 & 0,03 & $0,8-1,5$ & 1,2 & 0,04 \\
\hline Rapport $\frac{\text { ventouse orale }}{\text { pharynx }}$ & $1,3-1,9$ & 1,7 & 0,05 & $1,4-1,8$ & 1,6 & 0,03 \\
\hline
\end{tabular}

les deux lignées, ne sont pas significatives au seuil de $1 \%$ (tableau VI). La comparaison de deux échantillons de même âge, l'un appartenant à la lignée S. mutabilis, l'autre à la lignée $A$. corniculum, révèle une assez remarquable similitude (tableau VII; fig. 7 et 8).

\section{IV - Comparaison des caractères biologiques}

Nous comparerons les principaux caractères biologiques propres à chaque stade du cycle de vie, dans les deux lignées de parasites.

\section{A - Les rédies}

Les petites et les grandes rédies sont mélangées; elles envahissent d'abord la glande génitale puis la totalité de la glande digestive. Cette dernière est contiguë à l'espace s'étendant autour et sur toute la longueur du rectum. Au fur et à mesure que la maturation des parasites progresse, cet espace périrectal s'hypertrophie et finalement devient très volumineux. Il renferme en effet de nombreuses rédies qui sont souvent disposées parallèlement les unes aux autres et à l'axe du rectum. 


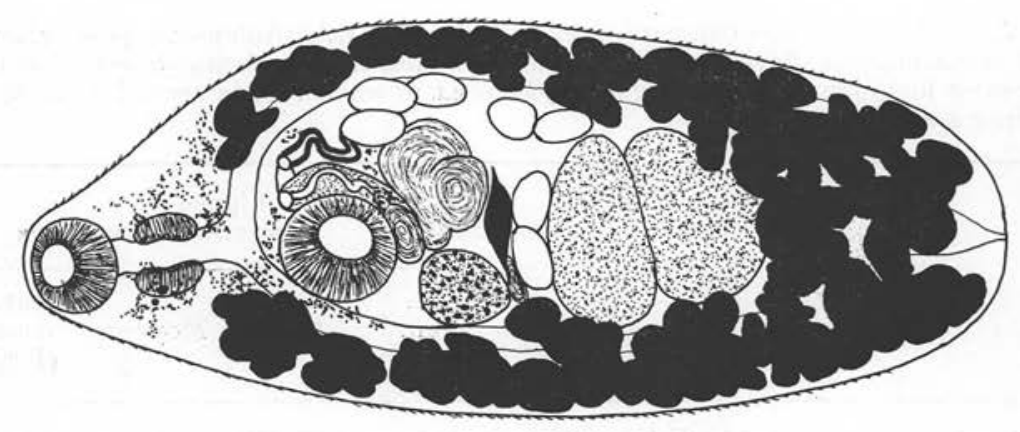

औ̊.
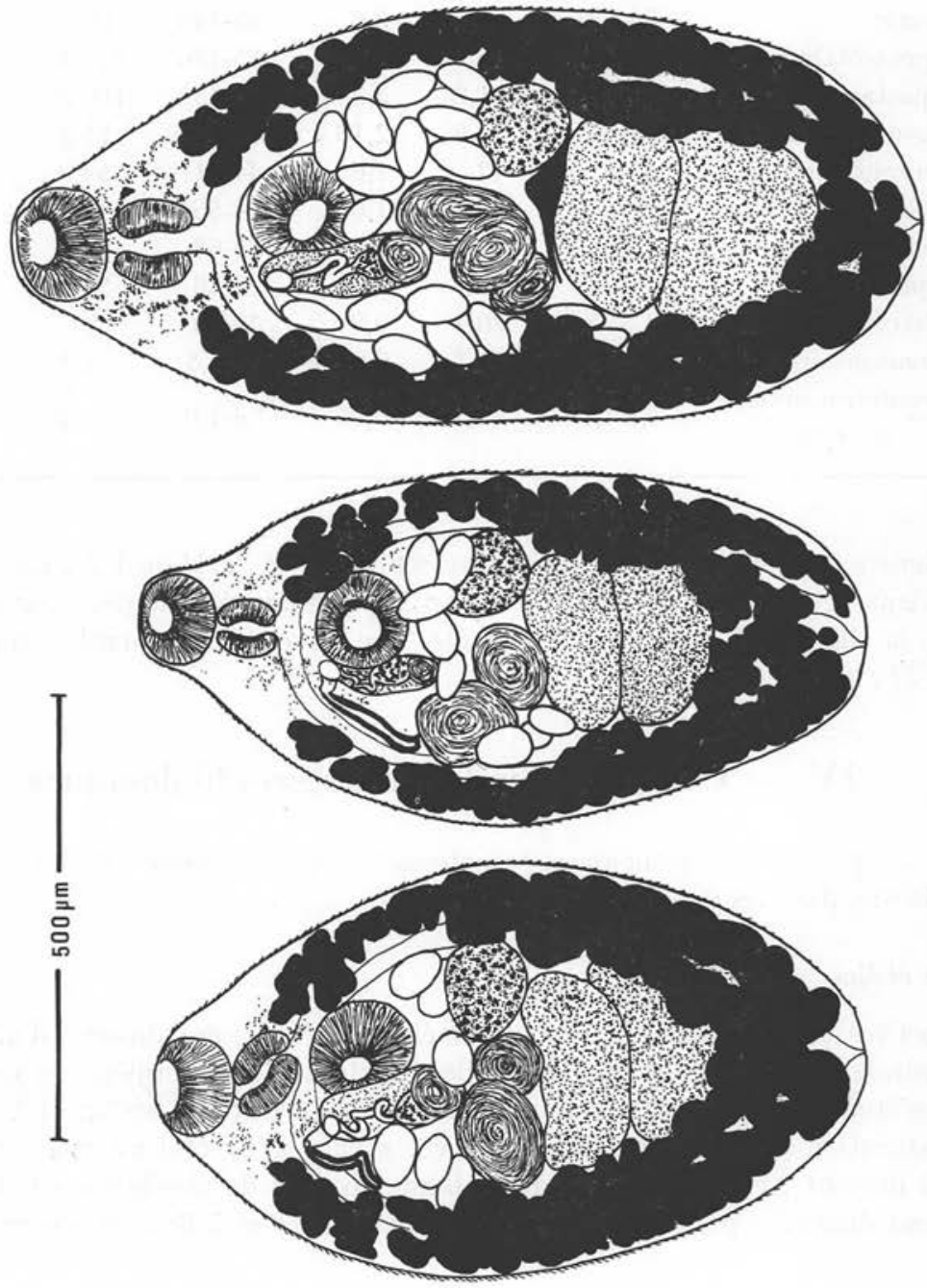


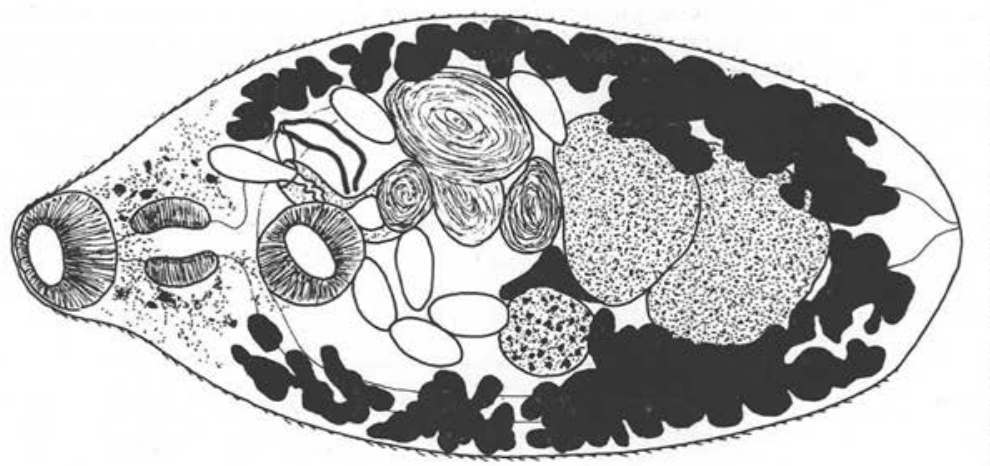

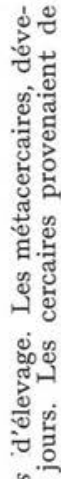

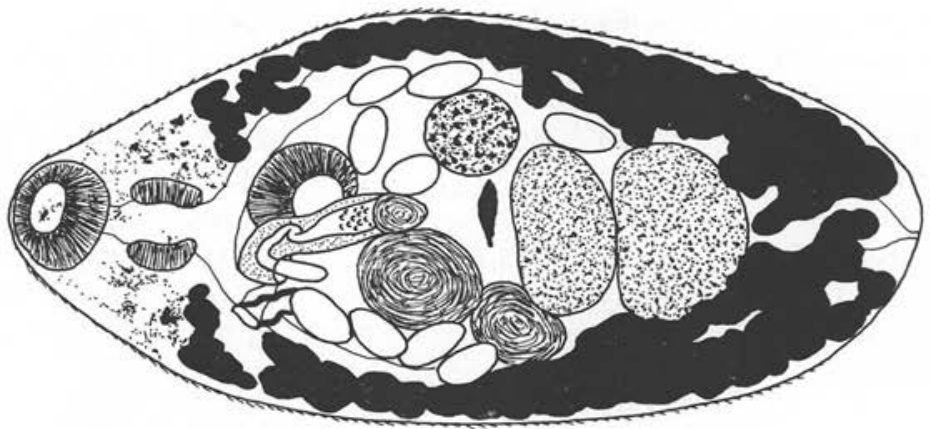

ริำ

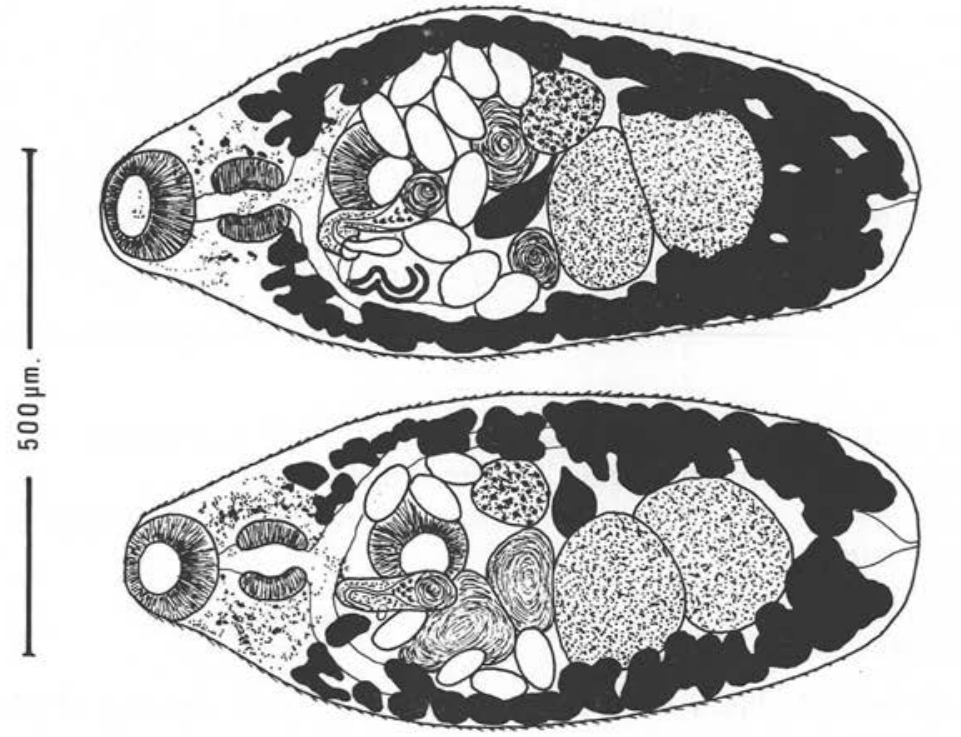

क्ष

है.

을

$\infty$ శ్

ขै है

为

तై

$\times$

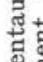

블 밍

폴

들

s.․ㄹ

है

s.

$\infty$ 으

它 
TABleau VI. - Mensurations comparées (en $\mu \mathrm{m}$ ) de deux lots composés chacun de 50 adultes développés expérimentalement chez Sparus auratus; le premier appartient à la lignée Sphaeronassa mutabilis, le second à la lignée Amyclina corniculum.

\begin{tabular}{|c|c|c|c|c|c|c|}
\hline & \multicolumn{3}{|c|}{$\begin{array}{c}\text { Lignée Sphaeronassa } \\
\text { mutabilis }\end{array}$} & \multicolumn{3}{|c|}{$\begin{array}{l}\text { Lignée Amyclina } \\
\text { corniculum }\end{array}$} \\
\hline & $\begin{array}{l}\text { Varia- } \\
\text { tions }\end{array}$ & Moyenne & $\begin{array}{l}\text { Int. } \\
\text { conf. } \\
(1 \%)\end{array}$ & $\begin{array}{l}\text { Varia- } \\
\text { tions }\end{array}$ & Moyenne & $\begin{array}{l}\text { Int. } \\
\text { conf. } \\
(1 \%)\end{array}$ \\
\hline Longueur totale & $480-1162$ & 778 & 57,09 & $506-1285$ & 777,5 & 68,8 \\
\hline Largeur totale & $240-533$ & 367,3 & 23,1 & $235-480$ & 325,2 & 25,4 \\
\hline Longueur préacétabulaire & $112-266$ & 195,7 & 12,6 & $91-373$ & 232,8 & 19,3 \\
\hline Longueur postacétabulaire & 261-784 & 482,4 & 41,4 & $277-800$ & 451,3 & 47,9 \\
\hline Longueur ventouse orale & $63-105$ & 81,7 & 3,3 & $70-126$ & 93 & 5,5 \\
\hline Largeur ventouse orale & $70-126$ & 97,9 & 3,1 & $78-144$ & 108,2 & 5,9 \\
\hline Longueur ventouse ventrale & $73-128$ & 100,2 & 3,7 & $63-122$ & 94,9 & 5,3 \\
\hline Largeur ventouse ventrale & $77-119$ & 95,7 & 4,1 & $63-125$ & 93,7 & 4,7 \\
\hline Longueur du pharynx & $49-89$ & 68,7 & 3,6 & $56-105$ & 76,6 & 4,7 \\
\hline Largeur du pharynx & $42-89$ & 65,7 & 3,9 & $45-115$ & 73,8 & 6,7 \\
\hline Longueur testicule antérieur & 56-154 & 92 & 9,6 & $49-168$ & 92,6 & 12,8 \\
\hline Largeur testicule antérieur & $98-230$ & 166 & 11,3 & $91-238$ & 151,3 & 13,1 \\
\hline $\begin{array}{l}\text { Longueur testicule } \\
\text { postérieur }\end{array}$ & $60-175$ & 105 & 10,2 & $70-175$ & 105,2 & 9,7 \\
\hline Largeur testicule postérieur & $98-238$ & 165 & 11,8 & $108-259$ & 159 & 14,8 \\
\hline Longueur ovaire & $56-126$ & 96,7 & 5,6 & $49-112$ & 73,1 & 5,6 \\
\hline Largeur ovaire & $49-113$ & 72,9 & 5,5 & $42-95$ & 64 & 5 \\
\hline Nombre d'œufs & $1-18$ & 8,4 & 1,3 & $1-21$ & 7 & 1,8 \\
\hline Longueur des œufs & $61-84$ & 75,2 & 1,3 & $63-91$ & 75,4 & 1,4 \\
\hline Largeur des œufs & $28-44$ & 36,5 & 0,9 & $28-54$ & 35,8 & 1,2 \\
\hline Rapport ventousaire & $0,68-1,04$ & 0,82 & 0,03 & $0,70-1,33$ & 0,98 & 0,03 \\
\hline Rapport $\frac{\text { ventouse orale }}{\text { pharynx }}$ & $0,95-1,42$ & 1,19 & 0,03 & $0,87-1,44$ & 1,19 & 0,02 \\
\hline
\end{tabular}

Les rédies sont logées dans les sinus hémaux; elles sont peu actives.

Le parasitisme s'accompagne invariablement d'une castration.

Tous ces caractères sont propres aussi bien aux parasites de $S$. mutabilis qu'à ceux d'A. corniculum.

\section{B - Les cercaires}

Les caractères biologiques du stade cercaire sont rigoureusement identiques dans les deux lignées. 
Tableau VII. - Dimensions (en $\mu \mathrm{m}$ ) comparées de deux échantillons d'adultes âgés de 28 jours, développés expérimentalement chez Sparus auratus. Le premier échantillon (4 individus) appartient à la lignée Sphaeronassa mutabilis, le second (5 individus) à la lignée Amyclina corniculum.

\begin{tabular}{|c|c|c|c|c|}
\hline & \multicolumn{2}{|c|}{$\begin{array}{l}\text { Lignée issue de } \\
\text { S. mutabilis }\end{array}$} & \multicolumn{2}{|c|}{$\begin{array}{l}\text { Lignée issue de } \\
\text { A. corniculum }\end{array}$} \\
\hline & Variations & Moyenne & Variations & Moyenne \\
\hline Longueur totale & 778-1 039 & 908 & $783-922$ & 849 \\
\hline Largeur totale & $390-469$ & 432 & $325-431$ & 377 \\
\hline Longueur préacétabulaire & $171-266$ & 222 & $224-237$ & 231 \\
\hline Longueur postacétabulaire & $501-640$ & 568 & 447-592 & 515 \\
\hline Longueur ventouse orale & $76-100$ & 86 & $82-93$ & 86 \\
\hline Largeur ventouse orale & $100-126$ & 108 & $104-117$ & 109 \\
\hline Longueur ventouse ventrale & $108-128$ & 116 & $97-108$ & 107 \\
\hline Largeur ventouse ventrale & $108-119$ & 114 & $91-106$ & 100 \\
\hline Longueur pharynx & $69-89$ & 77 & $67-78$ & 70 \\
\hline Largeur pharynx & $74-89$ & 80 & $78-89$ & 83 \\
\hline Longueur testicule antérieur & $98-135$ & 119 & $100-141$ & 122 \\
\hline Largeur testicule antérieur & $190-230$ & 208 & $152-195$ & 166 \\
\hline Longueur testicule postérieur & $98-174$ & 124 & $119-140$ & 128 \\
\hline Largeur testicule postérieur & $170-220$ & 192 & $158-195$ & 181 \\
\hline Longueur ovaire & $91-108$ & 98 & $74-98$ & 86 \\
\hline Largeur ovaire & $82-113$ & 95 & $67-87$ & 79 \\
\hline Longueur des œufs & $72-73$ & 72 & $72-76$ & 73 \\
\hline Largeur des œufs & $39-46$ & 43 & $39-54$ & 44 \\
\hline Rapport ventousaire & $0,67-0,85$ & 0,73 & $0,77-0,88$ & 0,83 \\
\hline Rapport $\frac{\text { ventouse orale }}{\text { pharynx }}$ & $0,97-1,20$ & 1,12 & $1,19-1,28$ & 1,23 \\
\hline
\end{tabular}

Maturation. La maturation des cercaires s'effectue à l'extérieur des rédies. Les cercaires sont libérées dans l'hémocoele dans un état très peu avancé. A ce moment là, les taches oculaires ne sont pas pigmentées; la constriction séparant le corps de la queue est seulement ébauchée ; les soies n'existent pas ; le tube digestif n'est pas formé. Ces cercaires très immatures sont immobiles.

Les soies caudales apparaissent peu de temps avant l'émergence des cercaires. Tous les stades de l'évolution des cercaires sont visibles dans l'hémocoele de l'hôte. Les cercaires mûres sont très actives; on les observe un peu partout, dans la glande génitale aussi bien que dans la glande digestive ou l'espace périrectal.

Concentration précédant l'émission. A partir du moment où commencent les émissions de cercaires, il s'en produit une chaque jour. Lorsque l'émission quotidienne 
est terminée, l'espace périrectal se trouve alors vidé des cercaires mûres qu'il renfermait. Des cercaires pleinement achevées gagnent alors petit à petit l'espace périrectal dans lequel elles sont de plus en plus nombreuses ; elles proviennent des sinus hémaux de la gonade ou de la glande digestive. Quelques instants avant le début de l'émission, le plus grand nombre de cercaires est observé au voisinage de l'anus. Ces cercaires sont extrêmement actives; leur queue est animée de mouvements incessants.

Émission. La présence d'une grande quantité de cercaires dans l'espace périrectal a pour conséquence une distension des parois que le circonscrivent. Cette membrane est fine; elle se déforme sous l'effet des mouvements des cercaires, en particulier sous l'action de la succion ventousaire. Finalement se produit une petite déchirure : une cercaire s'y engage alors. A l'aide de sa ventouse orale et par une succession de plusieurs mouvements d'élongation et de contraction, la cercaire réussit à agrandir la fissure et à percer la paroi. C'est à travers ce trou que s'échapperont les autres cercaires. Le lendemain, un autre orifice devra être réalisé.

Après effraction de la paroi de l'espace périrectal, les cercaires parviennent dans la cavité palléale ; elles sont ensuite entraînées à l'extérieur par le courant respiratoire.

Les émissions de cercaires sont quotidiennes, massives et limitées dans le temps (fig. 9). En moyenne, l'essaimage commence entre 4 heures et 5 heures après le lever du soleil ; 3 heures plus tard, il est terminé. En été par exemple, les émissions débutent entre 9 heures et 10 heures; elles sont achevées à 12 heures. L'acrophase se situe autour de 10 heures.

Le synchronisme des émissions de cercaires par S. mutabilis et par A. corniculum est tout à fait remarquable. Seules diffèrent les quantités de cercaires émises. Ces différences tiennent au fait que les dimensions de $S$. mutabilis sont largement supérieures à celles de $A$. corniculum (cf. fig. 1).

Dans les quelques cas où les émissions de cercaires ont été étudiées chez les Lepocreadiinae, celles-ci se produisent la nuit ou tôt le matin (H. W. Stunkard 1969 b ; $1980 \mathrm{a}$ et b). Les cercaires quittent leur hôte en passant à travers les branchies (H. W. Stunkard 1980 a et b).

\section{Comportement}

La nage. La capacité natatoire varie considérablement avec le temps.

Les cercaires récemment émises par le Mollusque nagent vigoureusement. La trajectoire est tourbillonnante; son profil général est trés variable (fig. 10).

La cercaire monte ou descend activ əment. Les mouvements de la queue sont permanents. Au cours de la nage, la queue est maintenue rabattue contre la face ventrale du corps et celui-ci est généralement disposé au-dessous de la queue (fig. 10 a). La nage est interrompue dès que la cercaire rencontre l'interface; elle reprend quelques instants plus tard. Lorsque la cercaire touche le fond du récipient, les mouvements de la queue cessent aussitôt puis le corps se tord sur lui-même pour amener la ventouse orale en contact avec le substrat, comme si la larve cherchait à y pénétrer ( $f i g .10 \mathrm{~b})$. 


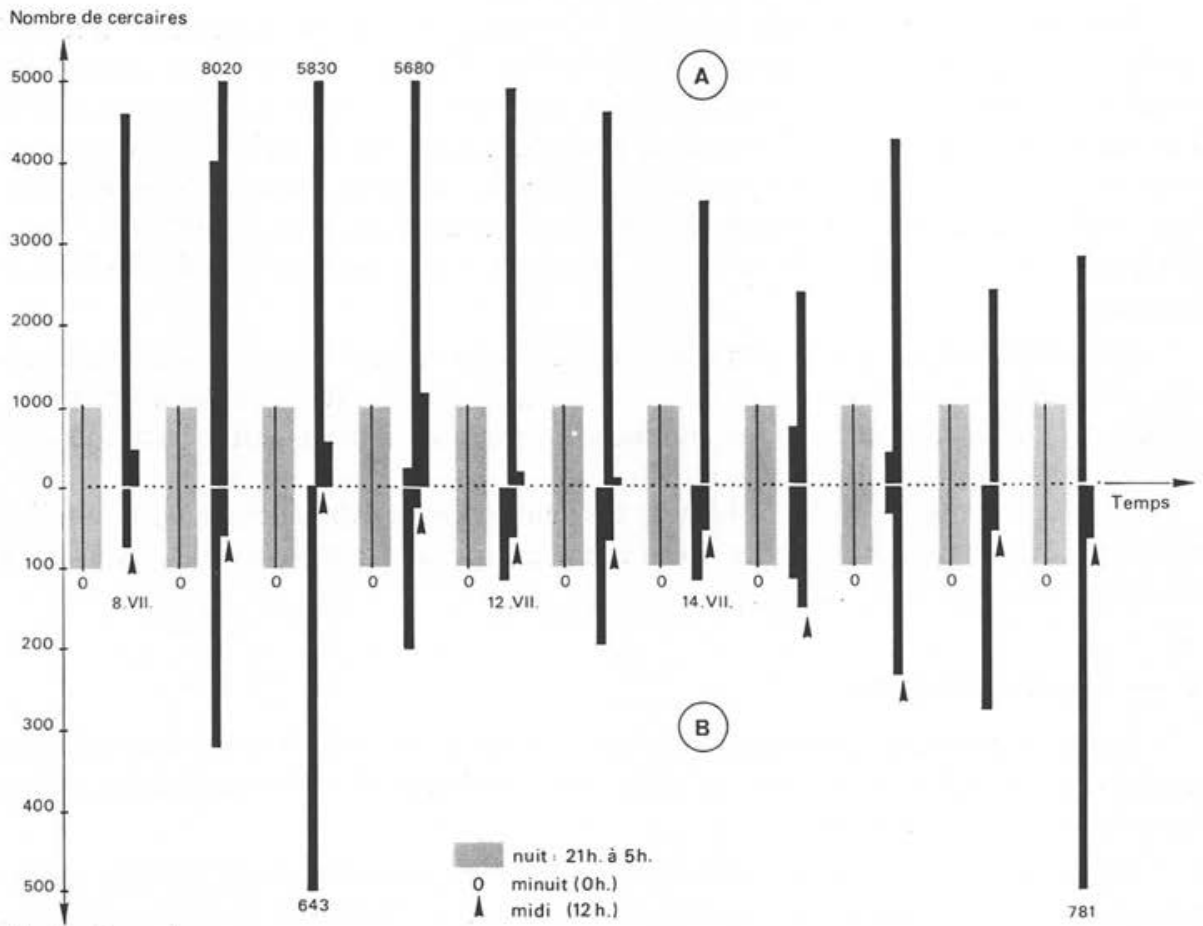

Nombre de cercaires.

FIG. 9. - Dénombrement des cercaires émises toutes les deux heures pendant une période de onze jours (Juillet 1982). A : cercaires issues de Sphaeronassa mutabilis; B : cercaires issues de Amyclina corniculum.

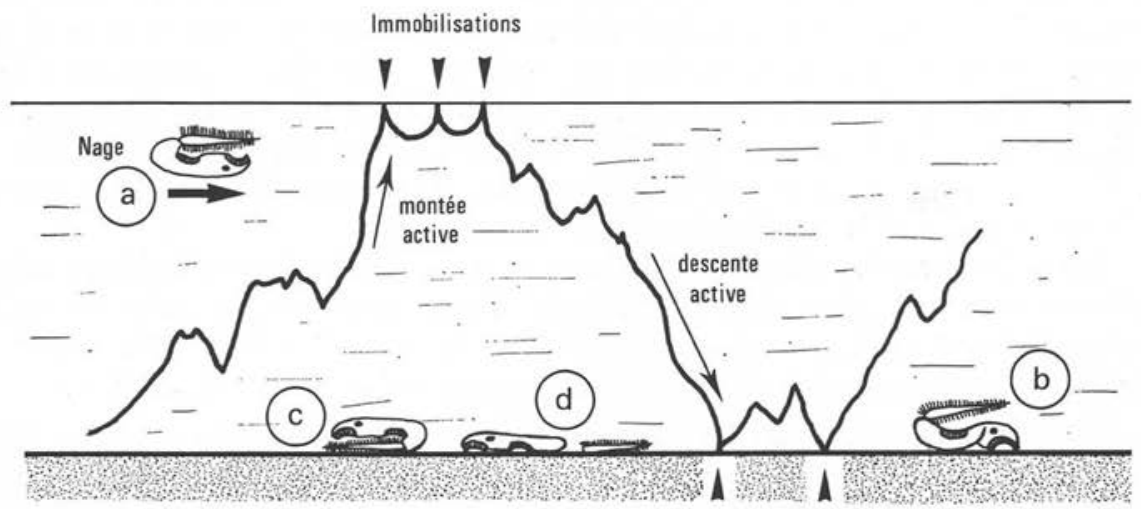

Immobilisation puis torsion du corps

FIg. Io. - Les cercaires essaimées par Sphaeronassa mutabilis et par Amyclina corniculum ont un même comportement. Le trait irrégulier schématise un fragment de la trajectoire suivie par une cercaire. a : attitude au cours de la nage; b : lorsque la cercaire entre en contact avec le substrat, la partie antérieure de son corps se tord sur elle-même. c, d : cercaires sorties du Mollusque émetteur depuis 24 heures; $c$ : la queue toujours présente s'étend sous le corps ; $\mathrm{d}$ : la queue est séparée du corps. 
Lorsque les cercaires sont âgées de 24 heures, près de $50 \%$ d'entre elles ont perdu leur queue ; plusieurs rampent alors sur le substrat ( $f i g .10 \mathrm{~d}$ ), d'autres sont immobiles. Lorsqu'on excite par une vive lumière les cercaires qui ont conservé leur queue, on constate qu'elles ne sont plus guère capables de s'élever au-dessus du fond du récipient malgré une grande agitation de la queue. Ces cercaires ont leur face ventrale tournée vers le substrat et reposent donc sur leur queue! ( fig. $10 \mathrm{c}$ ). Cette attitude est inverse de celle des cercaires récemment sorties du Mollusque émetteur.

Phototropisme. Le phototropisme est franchement positif chez les larves essaimées depuis peu de temps : les cercaires se rassemblent près de la lumière. Lorsqu'on modifie la localisation de la source lumineuse, les cercaires réagissent instantanément et vont se regrouper près de celle-ci.

Chez les larves âgées de 24 heures, toujours pourvues de leur queue, la lumière provoque une évidente excitation mais seule une petite fraction parvient à s'élever au-dessus du substrat et à nager.

\section{C - Les métacercaires}

Les métacercaires correspondant aux cercaires de Sphaeronassa mutabilis et à celles de Amyclina corniculum ne sont jamais enkystées ; elles sont pratiquement immobiles dans leurs hôtes.

Microhabitat. Chez leurs divers hôtes Lamellibranches, les deux types de métacercaires se rencontrent dans le même microhabitat préférentiel : les palpes labiaux. Beaucoup plus rarement on les retrouve dans la partie antérieure des branchies ou dans le manteau (microhabitats secondaires).

Hôtes expérimentaux. Les cercaires essaimées par $S$. mutabilis se développent en métacercaires chez Cerastoderma glaucum et Venerupis aurea; cette possibilité ne se réalise jamais dans la nature puisque ces Mollusques appartiennent à des écosystèmes différents. Inversement, les cercaires issues de $A$. corniculum sont capables d'évoluer en métacercaires chez Acanthocardia tuberculata, Parvicardium ovale, Venus gallina, Mactra corallina, Spisula subtruncata, Donax trunculus et D. semistriatus. D'autres Lamellibranches comme Abra ovata représentent des hôtes expérimentaux pour les métacercaires des deux lignées.

Lorsqu'on brise un exemplaire parasité de S.mutabilis, rédies et cercaires d'âges variés se répandent alors dans le récipient. On constate que les cercaires mûres pénètrent dans les rédies, comme s'il s'agissait là d'un hôte! Cette particularité tout à fait insolite s'observe aussi pour les cercaires et les rédies de A. corniculum.

Hôtes réfractaires. Tous les Lamellibranches ne représentent pas des hôtes possibles ; certains sont réfractaires. Dans l'endémiotope des sables vaseux de mode calme, Mytilus galloprovincialis, Venerupis (Tapes) decussata, Codakia decussata, Arcopagia balaustina, Notirus irus et Loripes lacteus ne sont jamais contaminés; leur infestation expérimentale par les cercaires issues aussi bien de Sphaeronassa mutabilis que de Amyclina corniculum, s'avère impossible. 
Les cercaires de $A$. corniculum pénètrent dans l'Opisthobranche Tectibranche Aplysia rosea (syn. A. punctata); ce Mollusque abonde dans l'herbier de Cymodocea nodosa. Cependant, les cercaires n'y évoluent pas en métacercaires : elles meurent rapidement. De même, les cercaires de Sphaeronassa mutabilis placées artificiellement en contact avec Aplysia rosea y pénètrent mais dégénèrent rapidement.

Les cercaires essaimées par Amyclina corniculum ne pénètrent pas dans le Coelentéré Parastephanauge pauxi (syn. Aiptasia mutabilis) présent en abondance sur les feuilles de Cymodocea nodosa. Les cercaires issues de Sphaeronassa mutabilis mises expérimentalement en présence de cet Actiniaire, n'y pénètrent pas non plus.

Maturation. Dans les deux lignées, les métacercaires deviennent infestantes au bout d'une quinzaine de jours environ.

\section{D - Les adultes}

Microhabitat. Les adultes de la lignée issue de $S$. mutabilis se répartissent préférentiellement dans les caecums pyloriques de leurs hôtes; quelques exemplaires s'installent dans le duodénum vraisemblablement lorsque la population est trop nombreuse dans les caecums pyloriques. Les adultes de la lignée A. corniculum occupent eux aussi les caecums pyloriques (microhabitat préférentiel) ; ils sont moins fréquents dans le duodénum (microhabitat secondaire) (tableau VIII).

TABleau VIII. - Infestations artificielles de Sparus auratus. Un premier Poisson a été contaminé par les métacercaires expérimentales de la lignée Sphaeronassa mutabilis, un second par les métacercaires expérimentales de la lignée Amyclina corniculum. Mise en évidence de la répartition des individus d'une population dans les différents microhabitats de leur hôte.

\begin{tabular}{|c|c|c|c|}
\hline & Parasite & $\begin{array}{l}\text { Caecums pyloriques } \\
\text { (microhabitat préférentiel) }\end{array}$ & $\begin{array}{c}\text { Duodénum } \\
\text { (microhabitat secondaire) }\end{array}$ \\
\hline $\begin{array}{l}\text { Poisson } \\
\mathrm{n}^{\circ} 1\end{array}$ & $\begin{array}{l}\text { lignée } \\
\text { S. mutabilis }\end{array}$ & $\begin{array}{l}108 \text { Adultes } \\
45 \text { Postlarves }\end{array}$ & $\begin{array}{l}25 \text { Adultes } \\
34 \text { Postlarves }\end{array}$ \\
\hline $\begin{array}{l}\text { Poisson } \\
n^{\circ} 2\end{array}$ & $\begin{array}{l}\text { lignée } \\
\text { A. corniculum }\end{array}$ & $\begin{array}{l}181 \text { Adultes } \\
62 \text { Postlarves }\end{array}$ & $\begin{array}{l}23 \text { Adultes } \\
22 \text { Postlarves }\end{array}$ \\
\hline
\end{tabular}

Maturation. Dans les deux lignées, les premiers œufs apparaissent une vingtaine de jours après la contamination.

Durée de vie. La durée de vie des adultes des deux lignées est comprise entre 35 jours et 40 jours. Dans les deux cas, la ponte des œufs s'effectue donc pendant une période d'une quinzaine de jours environ. 
Maturation et éclosion des aufs. Les œufs sont démersaux. Leur maturation est lente. Leur éclosion se situe en moyenne entre le $16^{\mathrm{e}}$ et le $21^{\mathrm{e}}$ jour ; ce phénomène est le même pour les œufs des adultes des deux lignées (fig. 11). Dans les deux cas, l'éclosion se produit toujours en fin de matinée, entre $6 \mathrm{~h}$ et $7 \mathrm{~h}$ après le lever du soleil. Les rendements des pontes et des éclosions semblent différents dans les deux lignées (tableau $I X)$.

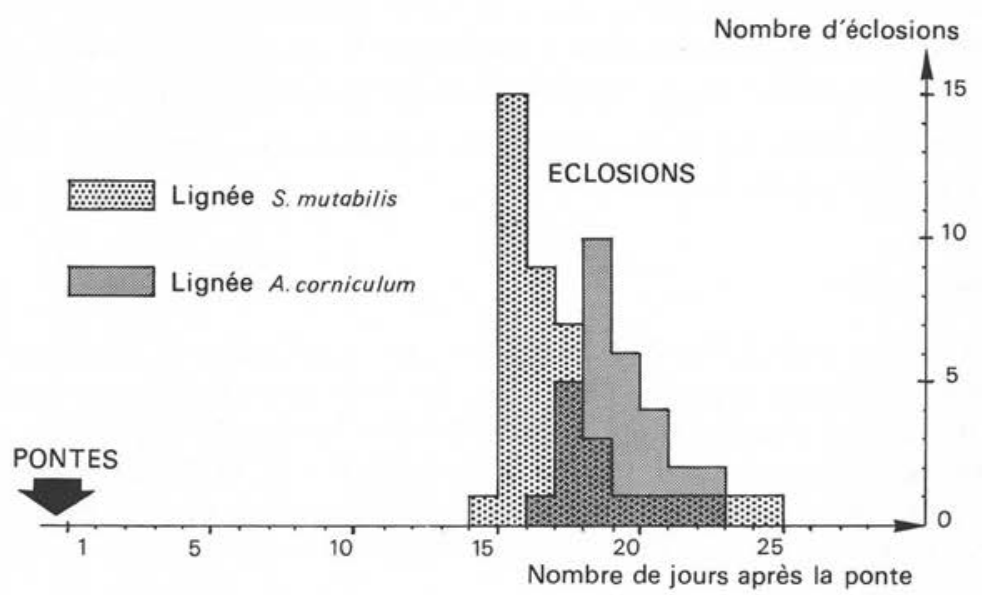

Fig. Ir. - Éclosions de deux lots d'œufs provenant, l'un d'adultes de la lignée issue de Sphaeronassa mutabilis, l'autre d'adultes de la lignée provenant de Amyclina corniculum.

TABleau IX. - Comparaison des pontes et des éclosions chez deux échantillons d'adultes expérimentaux âgés de 28 jours, développés expérimentalement chez Sparus auratus; le premier appartient à la lignée Sphaeronassa mutabilis (4 adultes), le second à la lignée Amyclina corniculum (5 adultes).

\begin{tabular}{lcc}
\hline & $\begin{array}{c}\text { Lignée } \\
\text { Sphaeronassa mutabilis }\end{array}$ & $\begin{array}{c}\text { Lignée } \\
\text { Amyclina corniculum }\end{array}$ \\
\hline Nombre de vers adultes & 4 & 5 \\
Nombre total d'œufs pondus en 24 h. & 98 & 36 \\
$\begin{array}{l}\text { Nombre moyen d'œufs pondus par } \\
\text { adulte en 24 h. }\end{array}$ & 24 & 7 \\
$\begin{array}{l}\text { Nombre d'œufs éclos } \\
\text { Nombre d'œufs morts }\end{array}$ & 41 & 30 \\
Nombre moyen de miracidiums pro- & 57 & 6 \\
duits par adulte en 24 heures & 10 & 6 \\
Rendement des éclosions & $41,8 \%$ & $83,3 \%$ \\
\hline
\end{tabular}




\section{Conclusions}

Tout au long de ce travail, nous nous sommes attachés à comparer, à chaque stade de leur cycle de vie, les deux lignées de ce Trématode. Plusieurs particularités les distinguent; d'autres au contraire leur sont communes.

1 - Les dissemblances. Les différences les plus évidentes sont relatives au cycle de vie. Les Mollusques premiers hôtes n'appartiennent pas à la même espèce. Cependant, bien que classés dans deux genres distincts, Sphaeronassa mutabilis et Amyclina corniculum sont apparentés. En effet, plusieurs auteurs considèrent que Sphaeronassa et Amyclina constituent deux sous-genres du genre Nassarius. D'ailleurs, la plupart des cercaires appartenant avec certitude aux Lepocreadiinae sont des parasites de Nassarius (M. Køie, 1975).

Les deuxièmes hôtes intermédiaires sont représentés par des espèces qui ne sont pas les mêmes dans les deux lignées.

D'autres différences importantes sont relevées dans les endémiotopes. Le premier correspond à un milieu très largement ouvert, le second à un milieu au contraire très confiné : ils appartiennent en fait à des écosystèmes diftérents. Toutefois, les Poissons hôtes définitifs, Sparus auratus, Pagellus erythrinus et P. mormyrus fréquentent les deux écosystèmes et passent facilement de l'un à l'autre.

Sur le plan biométrique, les dimensions de plusieurs organes des divers stades larvaires sont significativement différentes.

Enfin, au point de vue des caractères biologiques, certaines divergences apparaissent au niveau de la ponte (productivité) et de l'éclosion des œufs.

2 - Les similitudes. En ce qui concerne le cycle de vie, les hôtes définitifs de ces deux lignées sont les mêmes.

Sur le plan biométrique, les dimensions de la plupart des organes des vers adultes sont tout à fait comparables.

La quasi totalité des caractères morphologiques, anatomiques et biologiques sont identiques chez les divers stades de ces deux lignées.

Ce bilan comparatif n'apporte pas une réponse déterminante au problème posé et on ne peut affirmer s'il s'agit oui ou non de la même espèce. On en est donc réduit à des hypothèses ; il semble que l'on puisse en envisager trois :

- ou bien ces deux lignées constituent une seule et même espèce et l'on a affaire alors à deux populations ;

- ou bien les deux lignées constituent encore une seule et même espèce mais un début de spéciation à conduit à l'apparition de deux " races ";

- ou bien enfin un isolement reproductif a entraîné l'existence de deux espèces distinctes.

- Première hypothèse: les deux lignées sont une même espèce. On est alors en présence de deux populations distinctes. Ces deux populations sont bien isolées au début de leur cycle de vie puisque les premiers et les deuxièmes hôtes ainsi que les endémiotopes 
sont différents; par contre, elles se mélangent ensuite au niveau de l'hôte définitif car Sparus auratus, Pagellus erythrimus et $P$. mormyrus fréquentent les deux écosystèmes. Le brassage génétique est intense étant donné que la reproduction sexuée se réalise chez ces Poissons. Les différences observées sur le plan biométrique ne sont importantes que chez les stades larvaires; elles s'estompent chez les adultes. Ces dissemblances n'affectent pas le génotype mais seulement le phénotype ; elles sont dues à l'influence des hôtes intermédiaires qui ne sont pas les mêmes pour les deux lignées (l'hôte représente le milieu dans lequel se trouve le parasite).

En passant d'un écosystème à l'autre $S$. auratus, $P$. mormyrus et $P$. erythrinus sont parfaitement capables de contaminer deux milieux différents avec les œufs d'un même Trématode (fig. 12). La condition essentielle pour que le cycle puisse se réaliser consiste à trouver un premier hôte favorable. Dans le cas qui nous occupe, deux espèces apparentées mais caractéristiques des deux milieux ont été sélectionnées :

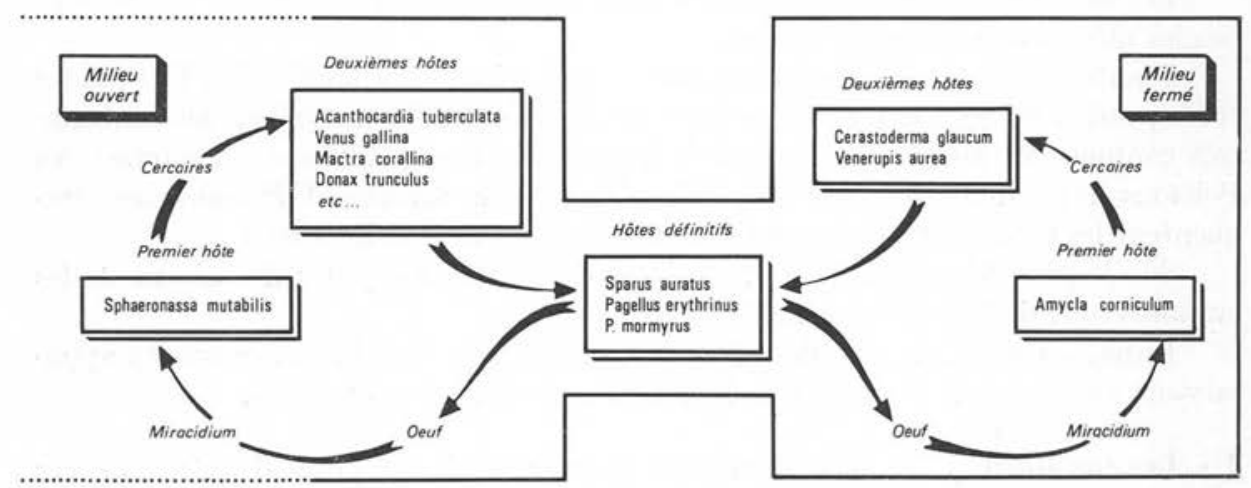

FIG. I2. - Hypothèse selon laquelle les deux lignées constituent une seule et même espèce. Le cycle biologique de Lepocreadium pegorchis se réaliserait dans deux écosystèmes différents (vicariance écologique) grâce aux Poissons hôtes définitifs qui passent de l'un à l'autre. Le premier hôte intermédiaire ne serait pas le même suivant qu'il s'agit de l'un ou de l'autre des deux endémiotopes; il en serait de même des deuxièmes hôtes intermédiaires.

Sphaeronassa mutabilis et Amyclina corniculum. Ces deux Mollusques deviennent donc des hôtes vicariants (sténoxénie). Le déroulement de la suite du cycle de vie ne pose alors aucune difficulté puisque d'une part, de nombreuses espèces de Lamellibranches peuvent constituer des hôtes favorables (euryxénie) et d'autre part, les Bivalves abondent toujours dans ces biocénoses. La sténoxénie au niveau du premier hôte intermédiaire, et l'euryxénie au niveau des deuxièmes hôtes ont ainsi permis la conquête de deux endémiotopes différents et nettement séparés, donc un accroissement considérable de l'aire de répartition du parasite. Si ces deux lignées s'avéraient représenter une seule et même espèce, on serait alors en présence d'un exemple tout à fait remarquable de vicariance écologique.

- Deuxième hypothèse : Les différences que l'on observe entre ces deux lignées affectent le génotype. Toutefois le stade de l'isolement reproductif biologique n'a 
pas encore été atteint. Aussi ces " races » se maintiennent-elles grâce à un écoulement de gènes de faible importance et cela, malgré les déplacements des Poissons d'un écosystème dans l'autre.

- Troisième hypothèse: Si ces deux lignées représentent deux espèces distinctes, il s'agit alors d'espèces jumelles. Dans ce cas, l'isolement des stades larvaires dans des milieux différents (c'est-à-dire dans des hôtes intermédiaires différents) a entraîné une dérive des pools géniques et finalement un isolement reproductif biologique. L'absence d'isolement spatial au niveau des stades adultes - vu que ceux-ci se répartissent dans le même microhabitat des mêmes hôtes définitifs - pose alors le problème délicat du mécanisme assurant l'isolement reproductif.

De ces trois hypothèses, la première paraît la plus vraisemblable. L'étude des iso-enzymes ou celle des infestations expérimentales croisées, pourrait apporter la réponse à ce problème. En ce qui concerne cette dernière méthode, et conformément au schéma expérimental proposé par L. Euzet et C. Combes (1980), nous avons tenté à diverses reprises des infestations artificielles des Mollusques premier hôte par les miracidiums des deux lignées; elles se sont malheureusement toujours soldées par des échecs. De nouveaux essais seront prochainement réalisés afin de donner une réponse définitive à ce problème.

\section{BIBLIOGRAPHIE}

Bartoli P. : Sur le cycle vital de Lepocreadium pegorchis (M. Stossich, I90o) M. Stossich, I903 (Trematoda, Digenea, Lepocreadiidae). C.R. Acad. Sc., 1966, 263, 1398-1400.

Bartoli P. : Étude du cycle évolutif d'un Trématode peu connu : Lepocreadium pegorchis (M. Stossich, 1900) (Trematoda, Digenea). Ann. Parasitol. Hum. Comp., 1967, 42, 605-6r9.

Euzet L., Combes C. : Les problèmes de l'espèce chez les animaux parasites. In : Les problèmes de l'espèce dans le règne animal (Ch. Bocquet, J. Génermont et Lamotte dir.). Mém. Soc. Zool. France, I980, I11, 239-285.

Køге M. : On the morphology and life-history of Opechona bacillaris (Molin, I859) Looss, I907 (Trematoda, Lepocreadiidae). Ophelia, 1975, 13, 63-86.

Mars P. : Recherches sur quelques étangs du littoral méditerranéen français et sur leurs faunes malacologiques. Vie et Milieu, 1966, 20, I-359.

Peres J. M., Picard J. : Nouveau manuel de bionomie benthique de la Mer Méditerranée. Rec. Trav. St Mar. End., 1964, 31, 5-137.

PICARD J. : Recherches qualitatives sur les biocoenoses marines des substrats meubles dragables de la région marseillaise. Rec. Trav. St. Mar. End., 1965, 36, I-160.

STUNKARD H. W. : Lepocreadium areolatum (Linton, I90o) n. comb. syn. Distomum areolatum Rudolphi of Linton, I9oo (Trematoda: Digenea). Trans. Amer. Microsc. Soc., I969 a, 88 , 79-84.

STUNKARD H. W. : The morphology and life history of Neopechona pyriforme (Linton, I9oo) n. gen., n. comb. (Trematoda : Lepocreadiidae). Biol. Bull., I969 b, 136, 96-I I3.

STUNKARD H. W. : Successive hosts and developmental stages in the life history of Neopechona cablei sp. n. (Trematoda : Lepocreadiidae). J. Parasitol., 1980 a, 66, 636-641.

STUNKARD H. W. : The morphology, life-history, and taxinomic relations of Lepocreadium areolatum (Linton, 1900) Stunkard, 1969 (Trematoda: Digenea). Biol. Bull., 1980 b, 158, 154-163. 